\title{
MICROSURGICAL ANATOMY AND INJURIES OF THE ABDUCENS NERVE
}

\author{
Flávio Ramalho Romero', Javier Gonzalez Ramos', Feres Chaddad-Neto², \\ Jose Manuel Almarcha Bethencourt ${ }^{7}$, Evandro de Oliveira ${ }^{3}$
}

The abducens nerve originates from a set of neural cells located in the ventral portion of the pons'. This cellular nucleus emits axons that attend a ventral course and leaves the brainstem in the pontomedullary sulcus ${ }^{2,3}$. The nerve has a previous anterior and superior trajectory, before it travel throught the dura-mater of the sellar region ${ }^{2}$. It passes throught the petrous portion of the temporal bone entering in the cavernous sinus ${ }^{4-8}$. The orbital curse begins in the common tendinous ring after it inervate the lateral rectus muscle ${ }^{9}$. Its course through the skull base is shown in Figures 1, 2 and 3.

The abducens is a somatomotor nerve whose function is contracting the lateral rectus muscle with consequent
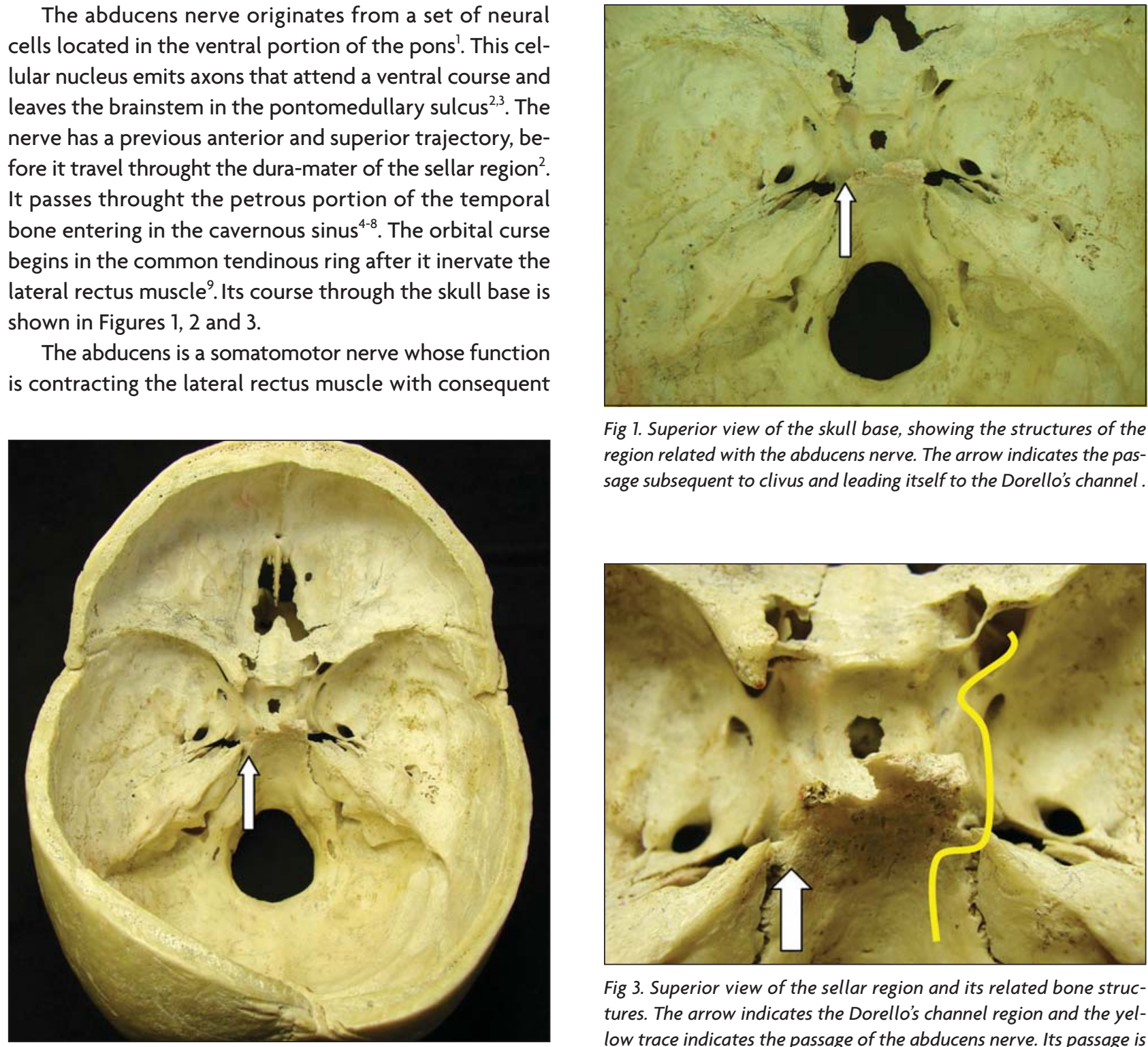

Fig 1. Superior view of the skull base, showing the structures of the region related with the abducens nerve. The arrow indicates the passage subsequent to clivus and leading itself to the Dorello's channel.

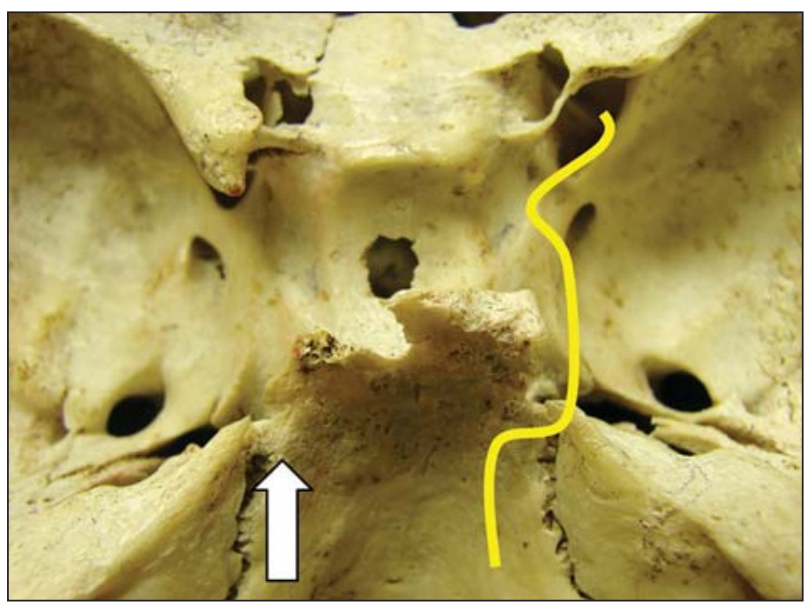

Fig 3. Superior view of the sellar region and its related bone structures. The arrow indicates the Dorello's channel region and the yellow trace indicates the passage of the abducens nerve. Its passage is subsequent to clivus in fossa posterior, passing the Dorello's channel, its intracavernous passage and the ticket for the superior orbital fissure to direct itself to the lateral straight muscle in orbit.

sage of the abducens nerve in the res leading itself to the Dorello's channel.

\section{ANATOMIA MICROCIRÚRGICA E LESÕES DO NERVO ABDUCENTE}

Instituto de Ciências Neurológicas (ICNE), Laboratório de Microcirurgia do Hospital da Beneficência Portuguesa de São Paulo, São Paulo SP, Brazil: ${ }^{1}$ Neurosurgeon Fellow of ICNE; ${ }^{2}$ Neurosurgeon of ICNE, Hospital Beneficência Portuguesa de São Paulo and Universidade de Campinas (UNICAMP); ${ }^{3}$ Director of ICNE, Chief of the Neurosurgery Division at UNICAMP.

Received 18 July 2008, received in final form 27 November 2008. Accepted 4 December 2008.

Dr. Feres Chaddad-Neto - Praça Amadeu Amaral 27 / 5? andar-01327-010 São Paulo SP-Brasil.E-mail:fereschaddad@hotmail.com; romerocnr@gmail.com 


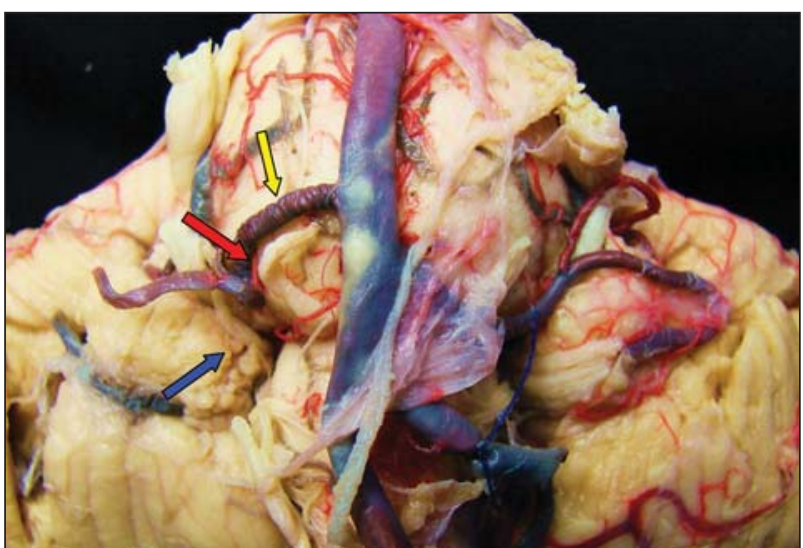

Fig 4. Previous view of the stem in the transition region between pons and medulla. The yellow arrow indicates the antero-inferior cerebelar artery, the red arrow shows the abducens nerve closely related to the AIC, and the blue arrow shows the facial nerve lateral to the abducens nerve in its origin at the ponto-medullary fissure.

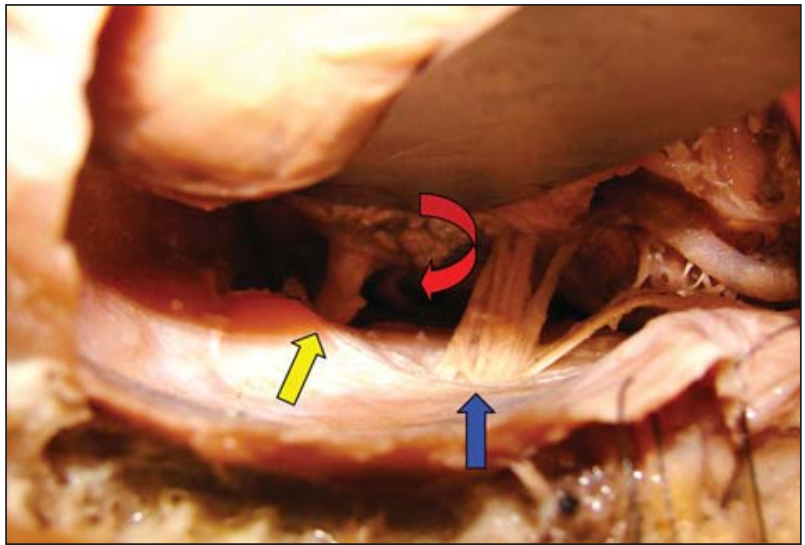

Fig 6. Retro-sigmoid access to the ponto-cerebelar angle region, showing the relation between the cranial nerves. The red arrow shows the abducens nerve, the yellow arrow points at the trigeminal nerve, and the blue arrow indicates the internal acoustic meatus region with the facial-intermediary and vestibule-coclear nerves.

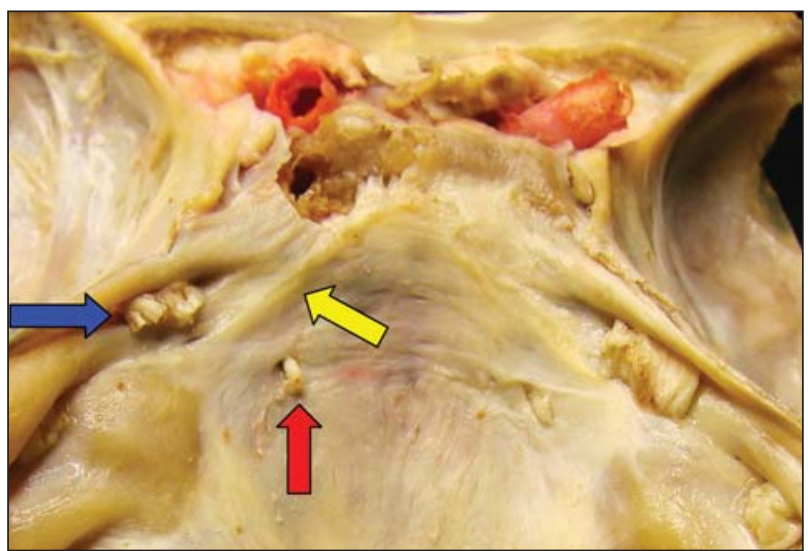

Fig 8. Posterior view of the petro-clival region. The yellow arrow indicates the Gruber's ligament. The blue arrow shows the trigeminal nerve and the red arrow points at the abducnes nerve leading itself through the Dorello's channel to the cavernous sinus.

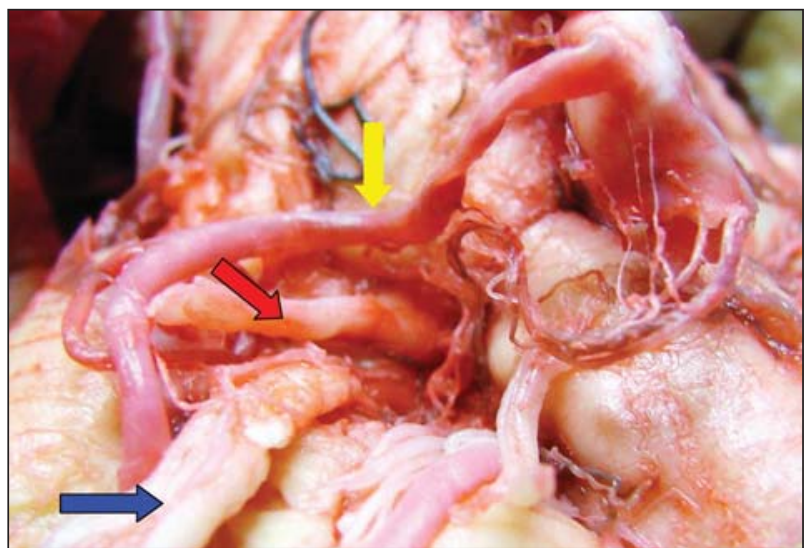

Fig 5. Antero-lateral view of the transition between pons and medulla. The yellow arrow shows the antero-inferior cerebelar artery. The red arrow points at the abducens nerve and the blue arrow shows the facial nerve.

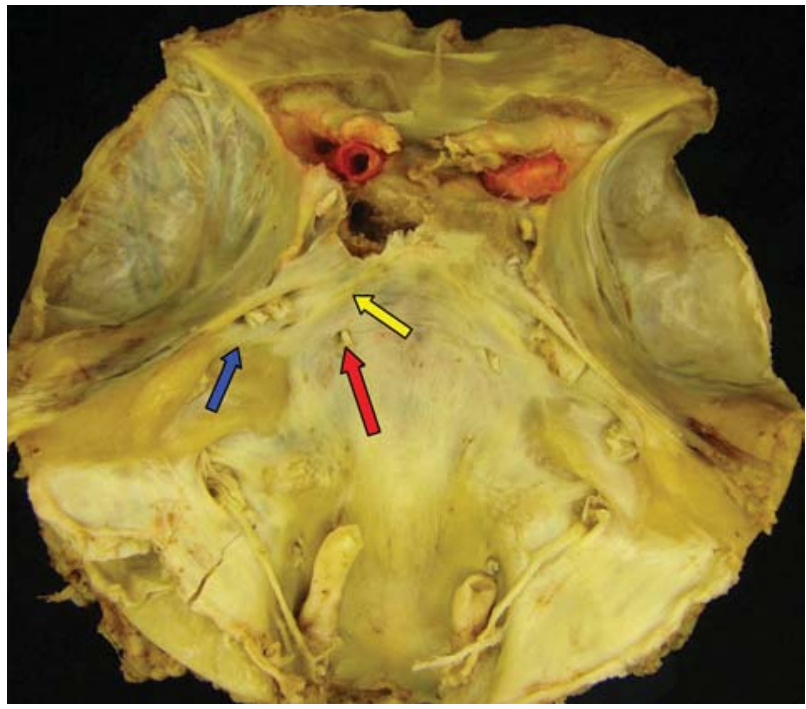

Fig 7. Posterior view of the skull base showing the relations between the cranial nerves and their origins. The yellow arrow indicates the Gruber's ligament, the red arrow shows the abducens nerve leading to the Dorello's channel and the blue arrow points at the trigeminal nerve.

eye abduction. It has a long passage from the brainstem to the ocular globe $e^{10-14}$.

We didactically cover in this study based on its location and relations to the central nervous system (CNS), posterior fossa, cavernous sinus and orbit. Also, we discuss some relative aspects regarding such nerve injuries.

\section{METHOD}

Twelve normal human cerebral hemispheres were fixed in a $10 \%$ formalin solution for over 40 days in preparation for the microsurgical dissection technique. The specimens were dissected with the operating microscope (X 6-40 magnification) in the stepwise manner. 
Five human heads were dissected to show the cavernous sinus region. The cavernous sinus was opened and its inner structures were exposed.

This study was approved by the local ethics committee.

\section{RESULTS}

\section{Posterior fossa (Figs 4-8)}

The abducens nerve is part of the middle neurovascular complex that includes the anterior inferior cerebellar artery (AICA), pons, middle cerebellar peduncle, cerebellopontine fissure, petrosal surface of the cerebellum, and the abducens, facial, and vestibulocochlear nerves ${ }^{3}$. It is closely related with these structures (Figs 4-6) and its anatomical and microsurgical knowledge becomes the basis for boarding situated injuries in this region such as tumors in the cerebellopontine angle and neurovascular

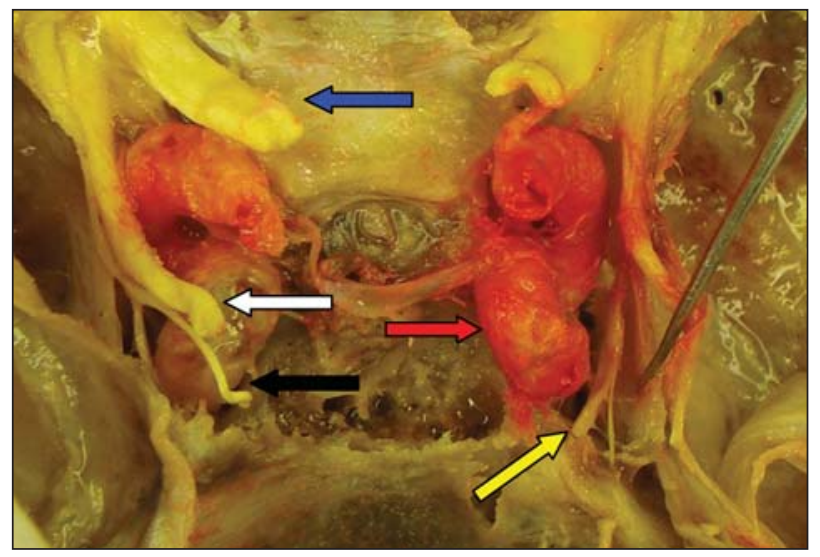

Fig 9. Posterior view of the left cavernous sinus, showing the neurovascular relations. The blue arrow shows the optic nerve, the white arrow points at the oculomotor nerve, the black arrow indicates the troclear nerve, the red arrow shows the intracavernous internal carotida artery and the yellow arrow points at the abducens nerve.

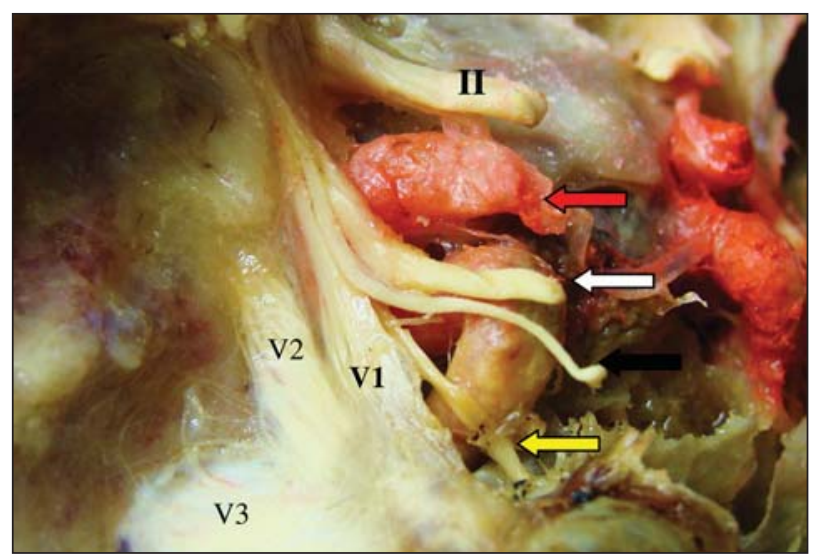

Fig 10. Postero-lateral view of the left cavernous sinus, showing the neuro-vascular relations. Observe the optic nerve (II) and the three divisions of the trigeminal nerve (V1, V2, V3). The red arrow points the internal carotida artery, the white arrow shows the oculomotor nerve, the black arrow indicates the troclear nerve, and the yellow arrow points at the abducens nerve. compressions associated with facial spasms and trigeminal neuralgia ${ }^{3,11}$.

The abducens nerve arises from the pontomedullary sulcus, medially to the facial nerve and the vestibulo-cochlear nerve (Figs 4-6). It penetrates the subarachnoid space in this portion traveling between the pons and clivus ${ }^{2}$.

It ascends adjacent to the anterior pontine membrane in the prepontine cistern which is the limit between the cerebellopontine cistern and prepontine cistern ${ }^{2,3}$. It penetrates the clival dura-mater and ascends to the cavernous sinus through the basilar sinus. In its way to the cavernous sinus, the abducens nerve passes through the Dorello's channel, situated between the petroclinoid ligament (Gruber's ligament) and the petrous bone apex (Figs 7-8) ${ }^{4-7}$.

The AICA arises at the pontine level and courses in relationship to the abducens, facial, and vestibulocochlear nerves to reach the surface of the middle cerebellar peduncle, where it courses along the cerebellopontine fissure and terminates by supplying the petrosal surface of the cerebellum. Operations directed to the middle complex are for the removal of acoustic neuromas and other tumors and for the relief of hemifacial spasm ${ }^{4-7}$.

The tentorium is attached to the petrous apex and to the anterior and posterior clinoid processes forming three dural folds or ligaments: the anterior petroclinoid ligament extending from the anterior clinoid process to the petrous apex, the posterior petroclinoid ligament extending from the posterior clinoid process to the petrous apex and the interclinoid ligament extending from the anterior to the posterior clinoid process. Such ligaments compose a triangular area in the posterior portion

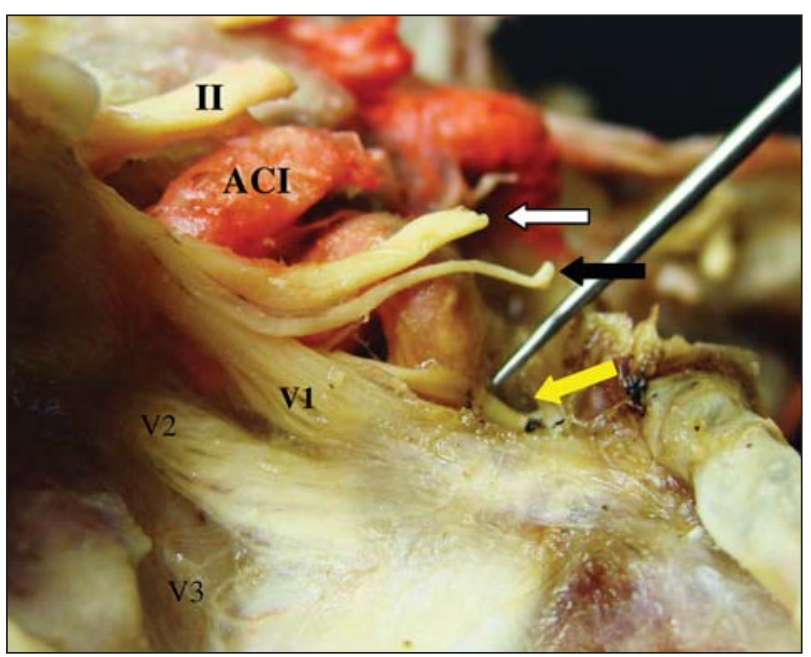

Fig 11. Lateral view of the left cavernous sinus, showing the neuro-vascular relations. The internal carotida artery is represented by the acronym $\mathrm{ACl}$ and the optic nerve by the II, while the trigeminal nerve divisions are with the initials V1, V2 and V3. The white arrow points at the oculomotor nerve, the black arrow indicates the troclear nerve and the yellow arrow shows the abducens nerve. 
on the roof of the cavernous sinus, the oculomotor triangle. The oculomotor nerve penetrates the dura in the central part of such triangle in order to reach the cavernous sinus (Figs 7-8) ${ }^{4-7}$.

\section{Cavernous sinus (Figs 7-11)}

The nerves in the sinus wall or sinus are, from superior to inferior, the IIIrd cranial nerve followed by the trochlear, ophthalmic, and abducens nerves. The oculomotor, trochlear, and ophthalmic nerves course in the inner part of the lateral sinus wall. The abducens courses medial to the ophthalmic nerve and is adherent to the lateral surface of the intracavernous carotid medially, but it also is adherent laterally to the medial surface of the ophthalmic nerve and the inner part of the lateral sinus wall (Figs 7-8) (-7 $^{4}$.

The abducens nerve pierces the dura forming the lower part of the posterior wall of the sinus at the upper border of the petrous apex and enters a dural cave, referred to as Dorello's canal, where it passes below the petrosphenoid ligament (Gruber's ligament) that extends from the lower part of the lateral edge of the dorsum sellae to the petrous apex, to enter the cavernous sinus (Figs 7-8) 4-7. $^{4}$.

The nerve bends laterally around the proximal portion of the intercavernous carotid and gently ascends as it passes forward inside the cavernous sinus medial to the ophthalmic nerve, on the lateral side of the internal carotid artery, and below and medial to the nasociliary nerve. It has the most medial site of entry of the nerves coursing in the sinus wall and maintains that position in its course through the sinus.

The nerve usually enters the sinus as a single bundle, but may persist as two bundles in the subarachnoid space. After entering the sinus, it may split into as many as five rootlets as it courses between the internal carotid artery and ophthalmic nerve. About $70 \%$ of the individuals present only one root, $25 \%$ two roots, $4 \%$ three roots and less than $1 \% 4$ or 5 roots ${ }^{4,7}$.

Inside the cavernous sinus, sympatic fibers that involve the internal carotid artery leave the artery and attend a course, first, with the abducens nerve, and, later, with the ophthalmic division of the trigeminal nerve $e^{4-7}$.

\section{Orbit (Figures 12-15)}

The abducens nerve travels forward in the cavernous sinus on the medial side of the ophthalmic nerve and shifts laterally below the nasociliary nerve as it passes through the superior orbital fissure and annular tendon to enter the medial surface of the lateral rectus muscle. At the apex of the orbit, the nasociliary nerve and the inferior division of the oculomotor nerve curve medially as the abducens nerve shifts laterally to enter the medial surface of the lateral rectus muscle. Some fibers of the carotid sympathetic plexus pass to and course within the abducens nerve in the cavernous sinus ${ }^{9}$ (Figs 12-13).

The oculomotor nerve enters the orbit by passing through the medial part of the fissure on the lateral surface of the optic strut. At the level of the posterior margin of the fissure, in the area medial to the trochlear and nasociliary nerves, the oculomotor nerve splits into superior and inferior divisions that course one above the other as they pass through the central sector of the fissure and the oculomotor foramen on the medial side of the branches of the ophthalmic nerve. The superior division of the oculomotor nerve enters the orbit below the attachment of the superior rectus muscle to the annular tendon and sends its branches upward lateral to the optic nerve to reach the lower surface of the superior rectus and levator muscles. The inferior division courses inferiorly and medially as it proceeds through the fissure on the medial side of the nasociliary and abducens nerves. At the orbital apex, it splits into three individual branches: two are directed forward to reach the inferior rectus and inferior oblique muscles, and one passes medially below the optic nerve to enter the medial rectus muscle. In addition, the branch to the inferior oblique muscle gives rise to the motor (parasympathetic) root to the ciliary ganglion. The parasympathetic fibers synapse in the ciliary ganglion, which gives rise to the short ciliary nerves that pierce the sclera to reach the ciliary body and iris ${ }^{9}$ (Figs 14-15).

The trochlear nerve courses in the lateral wall of the cavernous sinus below the oculomotor nerve and above the ophthalmic nerve. It passes through the upper edge of the narrow lateral part of the superior orbital fissure outside the annular tendon and passes medially above the frontal nerve and the levator muscle to reach the superior oblique muscle 9 (Figs 14-15).

The ophthalmic branch of the trigeminal nerve is the smallest of the three trigeminal divisions. It is inclined upward as it passes forward near the medial surface of the dura forming the lower part of the lateral wall of the cavernous sinus to reach the superior orbital fissure. It is flattened in the wall of the cavernous sinus, but at the superior orbital fissure, it takes on an oval configuration. The ophthalmic nerve splits into the lacrimal, frontal, and nasociliary nerves as it approaches the superior orbital fissure?

The ophthalmic artery usually arises just above the cavernous sinus from the medial half of the superior aspect of the anterior bend of the internal carotid artery 9 . Its origin is located under the medial part of the optic nerve, just behind the optic canal. In the optic canal, the ophthalmic artery courses within the optic sheath below the optic nerve and through the annular tendon. It exits the optic canal and penetrates the optic sheath to enter the orbital apex on the inferolateral aspect of the optic 


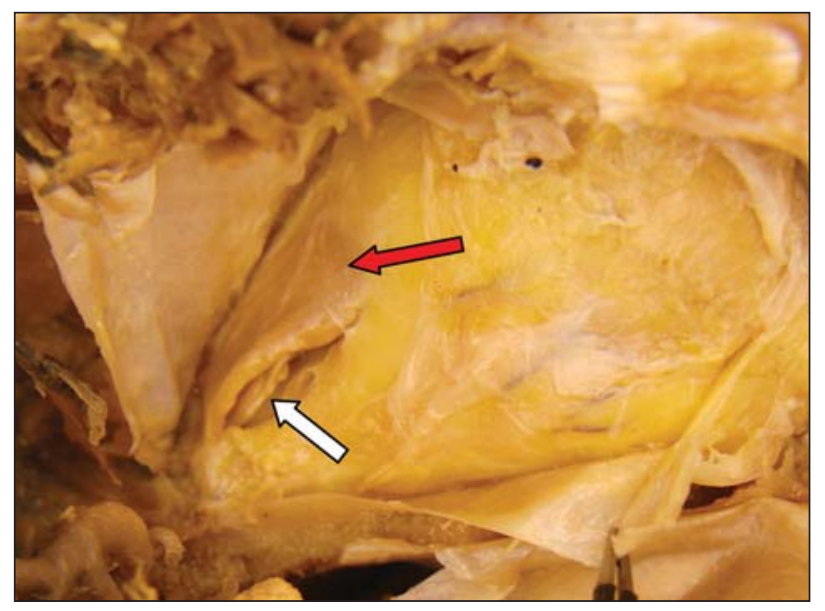

Fig 12. Superior view of the left orbit. The red arrow points at the lateral rectus muscle and the white arrow shows the abducens nerve.

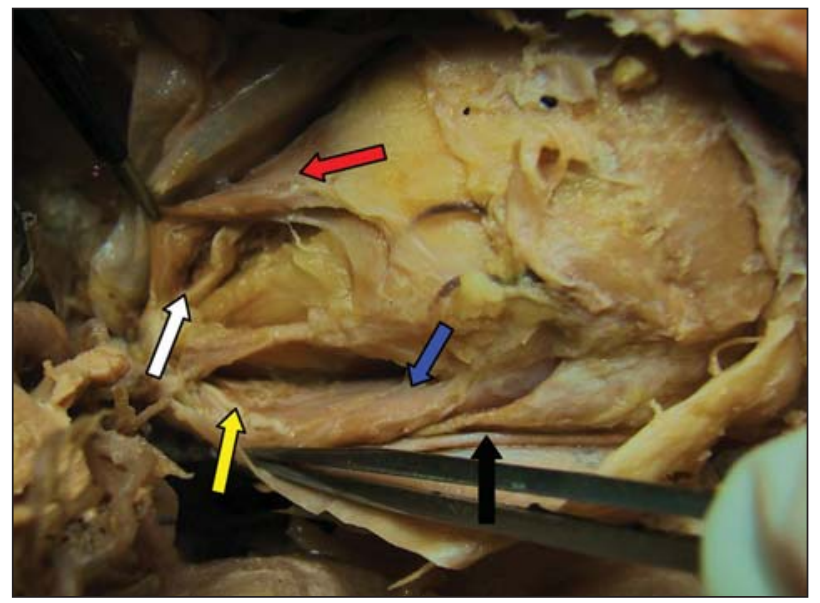

Fig 14. Superior view of the left orbit, showing the relations between nerves and muscles. The red arrow shows the lateral rectus muscle, the white arrow indicates the abducens nerve, the yellow arrow points at the oculomotor nerve superior branches, the blue arrow indicates the superior straight muscle and the black arrow points at the troclear nerve and the superior oblique muscle.

nerve. In the optic canal, the ophthalmic artery sometimes gives a recurrent branch to the intracranial segment of the optic nerve. Approximately $8 \%$ of ophthalmic arteries arise in the cavernous sinus rather than in the subarachnoid space?

\section{DISCUSSION}

The abducens nerve arises in a set of motor cells in the floor of the fourth ventricle, which are in the interior of the complicated handle formed for staple fibers of the facial nerve'. This motor nerve give rises to the lateral rectus muscle causing the abduction of the eye $\mathrm{e}^{1-3}$.

This thin nerve has a long intracranial passage, crosses the cavernous sinus and the superior orbital fissure to the

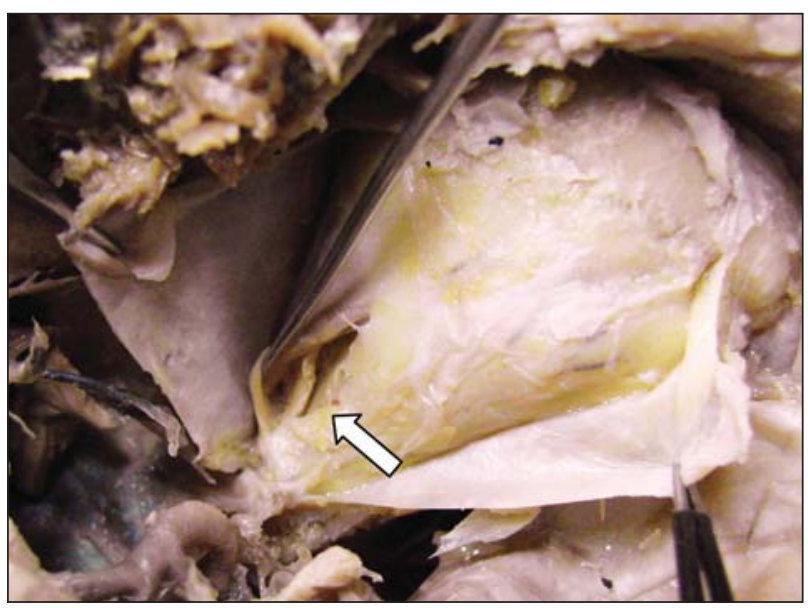

Fig 13. Superior view of the left orbit. The arrow points at the abducens nerve leading to the lateral straight muscle.

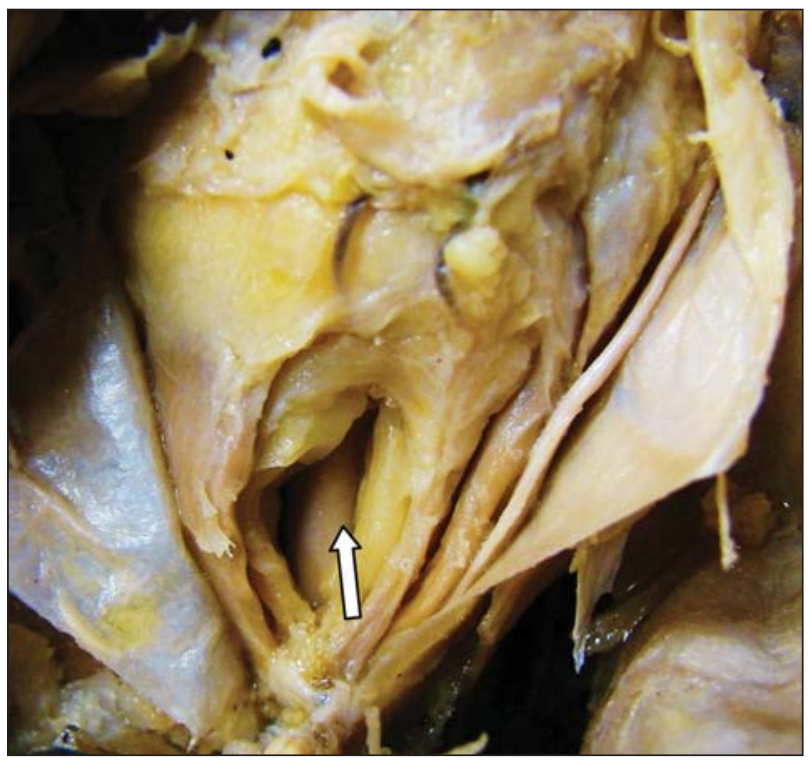

Fig 15. Superior view of the left orbit showing all the muscles up to the optic nerve identification, indicated by the arrow.

lateral rectus muscle. The abducens nerve is situated next to the internal carotid artery in the cavernous sinus ${ }^{4-7}$.

This cranial nerve nucleus is different from the other motor cranial nerve nuclei because it contains two neuronal populations: the typical motor neurons projecting staple fibers through the nervous root to make the ipsilateral rectus lateral muscle rise and the internuclears neurons, whose axons cross the middle line in the brainstem ending in the oculomotor complex cells to make the medial rectus muscle rise in the opposite side?.

The anatomical and microsurgical knowledge of the abducens nerve is essential in cases of ophtalmoplegias involving the eye lateral movement. As it has been described, we must be familiar with the pathologies that 
can cause any injuries to the abducens nerve, not only situated in the nuclei nerve but also located in the ventral portion of pons, the posterior fossa, the cavernous sinus and the orbit ${ }^{10-15}$.

About $8 \%$ to $30 \%$ of the cases are idiopatics, $3 \%$ to $30 \%$ related to trauma, $6 \%$ related to aneurysms, $36 \%$ of the cases related to ischemia, and $10 \%$ to $20 \%$ related to the infectious and inflammatory processes ${ }^{14}$ in the United States ${ }^{10}$.

The small unilateral injuries of the nerve abducens cause the lateral eye paralysis in the side of the injury. However, the lateral eye paralysis syndrome differs from the lateral rectus muscle paralysis because none of the eyes can be moved laterally to the side of the injury and both tend to forcibly deviate and in a conjugated way to the opposed side. The ocular convergence usually is not affected. Thus, the abducens nerve seems to be the only cranial nerve in which the radicular fiber injuries and the nucleus do not produce the same effect ${ }^{10,13}$.

The lateral eye paralysis provoked by the nucleus small injuries of the abducens nerve is caused by the motor neuron destruction of the nerve nucleus resulting in the ipsilateral straight muscle paralysis and the internuclear neurons destruction inside the abducens nerve nucleus and is the origin of the ascending fibers which project through opposing medial longitudinal fascicle to the medial rectum subdivision in the contra-lateral oculomotor complex ${ }^{10,12}$.

Not all the deficits in the lateral movement are associated with the abducens nerve paralysis. There are cases such as the orbital wall fracture, the Duane syndrome, miastenia gravis and the thyroid gland ${ }^{13}$ illnesses.

Amongst the main causes associated with the injuries in the passage from the ridge bulb-pontino up to the orbit are the intracranial hypertension which causes space alterations in the stem conformation and the Dorello's channel nerve compression. Around $30 \%$ of the abducens nerve paralysis cases are patients with pseudotumor cerebri ${ }^{14}$, the subarachnoid space injuries (hemorrhages, tumors, inflammation, infection and cavernous sinus tumors), inflammations, neoplasias of the pons, infections, trauma, vascular, metabolic and the idiopatic processes ${ }^{10-15}$.

In conclusion, the abducens nerve has a complex anatomy from its origin in the ventral portion of the pons and its relationship with the lateral straight muscle in the orbit. Injuries in the nervous system can be identified when associated with the anatomical knowledge and the complementary examinations. The microsurgical knowledge facilitates the approach and the treatment of neurosurgical injuries which are associated with deficits in the eye lateral movement.

\section{REFERENCES}

1. Carpenter MB, Carleton SC. Comparison of vestibular and abducens projections to the medial rectus subdivision of the oculomotor complex in the monkey. Brain Res 1983;274:144-149.

2. Liliquiest B. The anatomy oh the subaracnoid cisterns. Acta Radiol 1956; 46:61-71.

3. Martin RG, Grant JL, Peace DA, Theiss C, Rhoton AL Jr. Microsurgical relationship of the anterior inferior cerebellar artery and the facial vestibular nerve complex. Neurosurgery 1988;23:58-80.

4. De Oliveira E, Tedeschi E, Siqueira MG, Rhoton AL Jr. Microsurgical anatomy of the cavernous sinus. In: Salcman M (ed). Current techniques in neurosurgery, $2^{\text {nd }}$ Ed. Philadelphia: Current Medicine, 1996: 79-89.

5. Dolenc V. Direct microsurgical repair of intracavernous vascular lesion. J Neurosurg 1983;58:824-831.

6. Harris FS, Rhoton AL Jr. Anatomy of the cavernous sinus: a microsurgery study. J Neurosurg 1976;45:169-180.

7. Inoue T, Rhoton AL Jr, Theele D, Barry ME. Surgical approaches to the cavernous sinus: a microsurgical study. Neurosurgery 1990;26:903-932.

8. Natori Y, Rhoton AL Jr. Microsurgical anatomy of the superior orbital fissure. Neurosurgery 1995;36:762-775.

9. Al-Mefty O, Fox JL. Superolateral orbital exposure and reconstruction. Surg Neurol 1985;23:609-613.

10. Lana MA, Barbosa AS. Síndrome do seio cavernoso: estudo de 70 casos. Arq Bras Oftalmol 1998;61:635-639.

11. Koos WT, Spetzler RF, Lang J. Tumors of posterior skull base and craniocervical junction. Color atlas of microneurosurgery. Stuttgart: Thieme, 1993.

12. Gradenigro G. Surun syndrome particulier des complications endocraniennes otitiques. Arch Intern Laryng ol 1904;18:432.

13. Schapira AHV, Gale AN. Pseudomonas ostitis causing cranial nerve palsies. J Neurol Neirosurg Psychiatry 1985;48:1306-1307.

14. Friedman DI, Jacobson DM. Diagnostic criteria for idiopathic intracranial hypertension. Neurology 2002;59:1492-1495.

15. Rhoton AL Jr, Natori Y. The orbit and sellar region: microsurgical anatomy and operative approaches. New York: Thieme Medical, 1996:3-25. 